\title{
Supercritical water: Local order and molecular dynamics*
}

\author{
T. Tassaing ${ }^{\ddagger}$, Y. Danten, and M. Besnard \\ Laboratoire de Physico-Chimie Moléculaire, CNRS (UMR 5803), Université de \\ Bordeaux I, 351 Cours de la Libération, F-33405 Talence Cedex, France
}

\begin{abstract}
This paper is a study of the structure and dynamics of near-critical and supercritical water for thermodynamic states above the critical temperature in a wide range of density by infrared absorption and quasi-elastic neutron scattering. The evolution of the shape of the infrared profiles associated with the internal vibrational modes of water has been investigated. In supercritical water, at $T=380{ }^{\circ} \mathrm{C}$ and low pressure (density), in the range $25-50$ bar $\left(0.01-0.05 \mathrm{~g} \cdot \mathrm{cm}^{-3}\right)$, only monomers are detected. A progressive increase of the pressure (density) from 50 to $250 \mathrm{bar}$ (from 0.05 to $0.4 \mathrm{~g} \cdot \mathrm{cm}^{-3}$ ) leads to the appearance of dimers and trimers. In order to obtain information on the dynamics, we have performed incoherent quasielastic neutron-scattering experiments on light water for several thermodynamic states $\left(200<T<400{ }^{\circ} \mathrm{C}\right.$ and $184<P<400$ bar) corresponding to densities ranging from 0.2 to $0.9 \mathrm{~g} \cdot \mathrm{cm}^{-3}$. The results have been analyzed using a jump diffusion model and the two parameters of this model, namely, $\tau_{0}$, the residence time and $D$, the translational diffusion coefficient, have been determined as a function of the density.
\end{abstract}

\section{INTRODUCTION}

During the last decade, there has been an increasing interest in studies of the structure and dynamics of water at high temperatures and pressures and in particular in the supercritical domain. This can be explained by the fact that thermochemical properties of sub- and supercritical water (SCW) are remarkably different from those at ambient conditions [1-3]. The peculiar behavior of SCW is related to the changes in the hydrogen bond network at high temperatures. Thus, experimental investigations of the intermolecular structure and dynamics provide clues for the understanding of the SCW properties. The structural properties have been investigated using X-rays and neutron-scattering experiments [4-8]. It has been found that the number of hydrogen bonds per water molecule, which is about 3.5 in the liquid state, decreases to about 1.8 in dense supercritical water. In order to obtain information about the state of aggregation of water in a wide range of temperatures and pressures in the near-critical and supercritical domain, we have performed an investigation by infrared spectroscopy, which is particularly well adapted to probe hydrogen bonding even at very low densities. On the other hand, as incoherent neutron scattering has appeared to be a powerful tool to investigate the diffusive motions of water in the liquid state at low temperatures, this technique may be reliable for the investigation of the dynamics of supercritical water.

\footnotetext{
*Lecture presented at the European Molecular Liquids Group (EMLG) Annual Meeting on the Physical Chemistry of Liquids: Novel Approaches to the Structure, Dynamics of Liquids: Experiments, Theories, and Simulation, Rhodes, Greece, 7-15 September 2002. Other presentations are published in this issue, pp. 1-261.

${ }^{\ddagger}$ Corresponding author
} 


\section{EXPERIMENTAL DETAILS}

\section{Mid-infrared spectroscopy}

The measurements were performed on a Biorad interferometer (type FTS-60A) equipped with a globar source, a $\mathrm{KBr}-\mathrm{Ge}$ beam splitter and a DTGS detector. Single-beam spectra recorded in the spectral range $400-6000 \mathrm{~cm}^{-1}$ with a $2 \mathrm{~cm}^{-1}$ resolution were obtained by the Fourier transformation of $100 \mathrm{ac}-$ cumulated interferograms. We have used a special stainless steel cell designed in our laboratory in order to obtain a thin film of water at temperatures up to $380{ }^{\circ} \mathrm{C}$ and under pressures up to 250 bar. The sealing of the cell is ensured by a graphite ring, which is disposed around the window. The cell was equipped with two sapphire windows separated by a copper spacer ensuring a path length varying between 10 and $150 \mu \mathrm{m}$. Although the path length of the cell is known from the thickness of the spacer, we have usually used the interference technique to obtain an accurate determination of the thickness of the water layer. Let us emphasize that at least three different spacers, with thicknesses of 10, 25, and $150 \mu \mathrm{m}$, were needed to collect the data over the wide range of densities. We have checked the reproducibility of the data by measuring water under the same thermodynamics conditions with different path lengths. The heating of the cell was performed using a band heater disposed around the cell in which two thermocouples were placed, the first one was located close to the band heater to achieve the temperature regulation and the second was kept close to the sample area in order to measure the temperature of the sample with an accuracy of about $\Delta T \sim \pm 2 \mathrm{~K}$. The cell was then connected via a stainless steel capillary to a manual pump (Top Industrie SA), thus allowing to reach pressures up to $25 \mathrm{MPa}$ with an absolute uncertainty of $\pm 0.1 \mathrm{MPa}$ and a relative error of $\pm 0.3 \%$.

\section{Quasi-elastic incoherent neutron scattering}

The experiments have been performed using the time-of-flight spectrometer IN6 at the Institut Laue Langevin (ILL) in Grenoble (France) using an incident wavelength of $4.1 \AA$ with an energy resolution of about $160 \mu \mathrm{eV}$ in the momentum transfer range $(0.39<Q<2.5 \AA)$. For these high-temperature and -pressure measurements, we have used a sample cell made of niobium, for which the incoherent scattering length is zero. The cell is constituted of two concentric cylinders, the inner one with 6-mm diameter and the outer one with $6.2-\mathrm{mm}$ internal diameter and a wall thickness of $6 \mathrm{~mm}$. This geometry has been used in order to reduce the multiple scattering effects in water. The cell is connected via a long stainless steel capillary to a hydraulic pressurizing system that permits the control of the pressure up to $100 \mathrm{MPa}$ with an absolute uncertainty of $\pm 0.1 \mathrm{MPa}$ and a relative error of $\pm 0.3 \%$. The cell, the capillary, and the pressurizing system are filled with pure $\mathrm{H}_{2} \mathrm{O}$ at room temperature. The cell is placed in the center of a cylindrical vanadium-sheet furnace, which is regulated by a computerized temperature control $\left(\Delta T \sim \pm 0.5{ }^{\circ} \mathrm{C}\right)$. The measuring time of each spectrum varied from 8 to $16 \mathrm{~h}$ depending on the density of the water sample.

\section{RESULTS AND DISCUSSION}

\section{Mid-infrared absorption}

We have reported in Fig. 1 the evolution of the $\mathrm{OH}$ antisymmetric $\left(v_{3}\right)$ and symmetric $\left(v_{1}\right)$ stretches of SCW at $T=380{ }^{\circ} \mathrm{C}$ as a function of pressure (density) in the range $25-250 \mathrm{bar}\left(0.01-0.43 \mathrm{~g} \cdot \mathrm{cm}^{-3}\right)$. At the lowest density, we observe a remnant of the complicated rovibrational structure of the $v_{3}$ stretching mode centered at $3756 \mathrm{~cm}^{-1}$. As expected for a $C_{2 v}$ asymmetric top molecule, the $v_{3}$ mode exhibits a $\mathrm{P}, \mathrm{Q}, \mathrm{R}$ structure (A-type band).

Notice that the contribution of the $v_{1}$ mode at about $3657 \mathrm{~cm}^{-1}$ is not observed because its activity is lower by a factor of 10 compared with that of the $v_{3}$ mode [9]. The integrated intensity of absorption of water at $T=380^{\circ} \mathrm{C}$ and $P=25$ bar in the $\mathrm{OH}$ stretching region is about $59 \pm 2 \mathrm{~km} \mathrm{~mol}^{-1}$ 


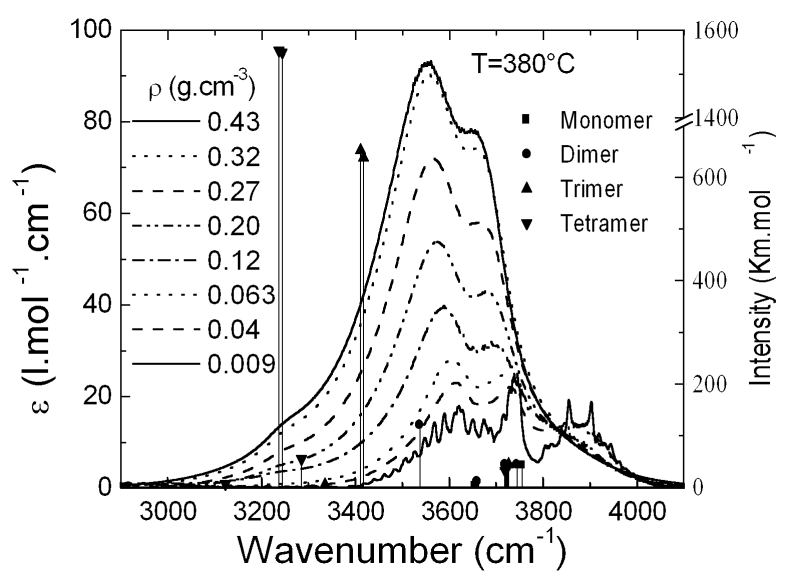

Fig. 1 Infrared spectrum in the $\mathrm{OH}$ stretching region of supercritical water at $T=380{ }^{\circ} \mathrm{C}$ as a function of pressure (density) in the range $25-250 \mathrm{bar}\left(0.01-0.4 \mathrm{~g} \cdot \mathrm{cm}^{-3}\right)$. The calculated characteristic frequencies of the $\mathrm{OH}$ stretching mode of small water clusters are reported for comparison (see text).

and found to be in almost perfect agreement with the value reported by Bondarenko et al. [10]. Therefore, under these conditions, water is in its monomeric form.

As the density increases from $\rho=0.01$ up to $\rho=0.1 \mathrm{~g} \cdot \mathrm{cm}^{-3}$, we observe an enhancement of the intensity of the spectrum and a significant perturbation of the band shape. This is reflected by a peak situated at about $3600 \mathrm{~cm}^{-1}$ superimposed over the envelop of the PQR structure, the Q branch of which is enhanced at about $3750 \mathrm{~cm}^{-1}$ comparatively to the wings whilst the $\mathrm{R}$ branch leads to a shoulder at $3850 \mathrm{~cm}^{-1}$. These spectral variations might be attributed to the occurrence of small oligomers in the fluid. Finally, in the density range $0.1<\rho<0.4 \mathrm{~g} \cdot \mathrm{cm}^{-3}$, we mostly observe a profile having a doublet structure. As the density increases, we observe a progressive enhancement of the intensity and a shift toward lower frequencies as well as a broadening of these profiles, leading to a broad band with two maxima at about 3650 and $3550 \mathrm{~cm}^{-1}$ at the highest density investigated. The shoulder observed at $3850 \mathrm{~cm}^{-1}$ at the lowest density progressively disappears, whereas a new shoulder is observed at about $3250 \mathrm{~cm}^{-1}$. This spectral evolution is in a good agreement with the spectral data reported by Gorbaty et al. [11] in the same density range but at a higher temperature, $T=500{ }^{\circ} \mathrm{C}$. In order to tentatively assign our IR experimental findings, we have also reported in Fig. 1 the computed IR activities at the frequency values of the stretching modes of monomeric water and small water clusters (ranging from dimer to tetramer) obtained from DFT calculations $[12,13]$. We observed in the region of the antisymmetric stretch, at about $3720-3750 \mathrm{~cm}^{-1}$, the band associated with the "free" $\mathrm{OH}$ stretching mode of the oligomers. It is found to be almost independent of the size of the cluster. In contrast, at lower frequencies, in the range $3200-3550 \mathrm{~cm}^{-1}$, we observe the peaks associated with $\mathrm{H}$-bonded $\mathrm{OH}$ stretches of the oligomers, the frequencies of which decrease strongly with the cluster size. Moreover, we observe a strong increase of the IR intensities with the cluster size. These spectral manifestations of the $\mathrm{OH}$ stretching band upon clustering are very helpful for the qualitative analysis of the IR spectra. Indeed, the peak centered at about $3600 \mathrm{~cm}^{-1}$ can be clearly associated with the $\mathrm{H}$-bonded $\mathrm{OH}$ stretch of a dimer, whereas the peak associated with trimers and tetramers is expected at lower frequency. The comparison of the calculated frequencies of the $\mathrm{OH}$ stretch with those of the experimental spectra allows us to infer that the $\mathrm{H}$-bonded species increase on going from density values of 0.01 up to $0.1 \mathrm{~g} \cdot \mathrm{cm}^{-3}$, and that they are essentially constituted of dimers. Therefore, only monomeric water is detected in SCW at $T=380{ }^{\circ} \mathrm{C}$ and low pressure (density) in the range $25-50 \mathrm{bar}\left(0.01-0.05 \mathrm{~g} \cdot \mathrm{cm}^{-3}\right)$. A progressive increase of the pressure (density) from 50 to 180 bar (from 0.05 to $0.1 \mathrm{~g} \cdot \mathrm{cm}^{-3}$ ) shows the occurrence of dimers in the fluid. These findings are in agreement with previously reported results $[10,14-16]$. In particular, we note that the density threshold from which dimers occur, at about 
$0.04-0.05 \mathrm{~g} \cdot \mathrm{cm}^{-3}$, is in qualitative agreement with other experimental [10] and theoretical work [14]. In addition, such a density threshold is also seen from NMR data [16] and molecular dynamics results [15] on rotational relaxation time.

Upon increasing the density from 0.1 to $0.4 \mathrm{~g} \cdot \mathrm{cm}^{-3}$, we can infer from the previous discussion that the $\mathrm{H}$-bonded species occurring in the fluid are mainly trimers, with the $\mathrm{OH}$ characteristic frequency of about $3410 \mathrm{~cm}^{-1}$. However, if tetramers are present in the fluid at higher densities, they are certainly in much lower proportion, as indicated by the weak shoulder at $3250 \mathrm{~cm}^{-1}$. This finding is supported by the fact that the IR activity of the characteristic mode of tetramers is strong so that even a small concentration of such clusters should give rise to a noticeable component. An alternative assignment of the shoulder observed at $3250 \mathrm{~cm}^{-1}$ may be the existence of a Fermi resonance between the overtone of the bending mode $\left(2 v_{2}\right)$ and the $\mathrm{OH}$ stretching mode, which has been put in evidence by Raman scattering [17].

Finally, the presence of larger clusters can be ruled out because their characteristic $\mathrm{OH}$ band is situated at lower frequencies than those observed here. Therefore, SCW is essentially composed of monomers, dimers, and trimers at $T=380{ }^{\circ} \mathrm{C}$ and pressures (density) in the range 200-250 bar $\left(0.1-0.4 \mathrm{~g} \cdot \mathrm{cm}^{-3}\right)$. This conclusion is consistent with molecular dynamics simulations, which provide estimates of the percentage of water molecules forming $i$ hydrogen bonds with their neighbors [18]. It was shown that under thermodynamics conditions close to ours, $i$ is ranging from 0 to 2 , that is to say, monomers, dimers, and trimers. Similar conclusions were reached by other authors from the analysis of molecular dynamics results in terms of H-bonded cluster size distributions [19].

\section{Quasi-elastic incoherent neutron scattering}

The quasi-elastic incoherent neutron-scattering spectra $I(Q, \omega)$ have been fitted using the equation:

$$
I(Q, \omega)=S(Q, \omega) \times R(\omega)+I_{\text {background }}
$$

where $R(\omega)$ is the instrumental resolution and $I_{\text {background }}$ denotes the background contribution. The incoherent scattering law $S(Q, \omega)$ is given by [20]:

$$
S(Q, \omega)=\exp \left(-Q^{2}\left\langle u^{2}\right\rangle / 3\right) T(Q, \omega) \times R(Q, \omega)
$$

where the first term is the Debye-Waller factor and $\left\langle u^{2}\right\rangle$ the mean-square amplitude of the vibrations. $R(Q, \omega)$ accounts for the hindered rotation and

$$
T(Q, \omega)=\frac{1}{\pi} \frac{\Gamma(Q)}{\omega^{2}+\Gamma(Q)^{2}}
$$

represents the contribution from the translational motions.

At this stage, we assume a priori that the rotational contribution is too fast to be detected here. A good fit of the experimental data has been obtained using eq. 1 and the evolution of the line widths $\Gamma(Q)$ as a function of $Q^{2}$ resulting from the fitting procedure are reported in Fig. 2 for three different densities. These curves display a linear increase at small $Q$ and tend toward an asymptotic value at higher $Q$ values. These features are characteristic of a jump diffusion process. In this case, the evolution of the line width $\Gamma(Q)$ as a function of $Q^{2}$ is given by:

$$
\Gamma(Q)=\frac{D Q^{2}}{1+D Q^{2} \tau_{0}}
$$

where $\tau_{0}$, is the residence time and $D$ the translational diffusion coefficient. An overall agreement is observed between the experimental and the fitted values (using eq. 4, see solid line in Fig. 2) of the line 


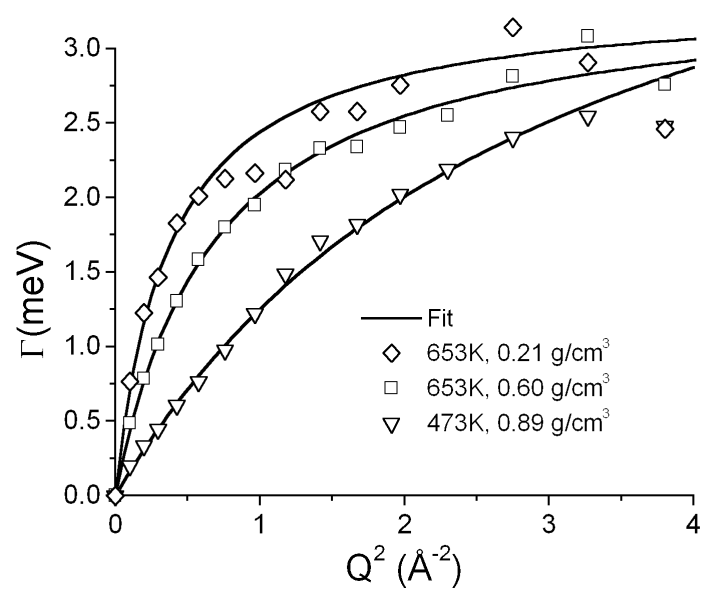

Fig. 2 Evolution of the linewidth $\Gamma(Q)$ of the quasi-elastic profile as a function of $Q^{2}$ for three different thermodynamics states of water. The full line is the result of the fit using the eq. 4 .

width as a function of $Q$. We notice some small discrepancies at high $Q$ values $\left(Q^{2}>3 \AA^{2}\right)$ and for the curve corresponding to the lowest density state because of the small signal-to-noise ratio of the quasielastic scattering in these two cases (cf. Fig. 2). Thus, from the good fit obtained with this model, we have determined the parameters $D, \tau_{0}$ and the mean jump distance $L$, which is related to $D$ and $\tau_{0}$ by $L=\sqrt{6 D \tau_{0}}$. The evolution of these three parameters as a function of the density of supercritical water is reported in Figs. 3-5, respectively. The corresponding error bars are due to the inherent signal to noise ratio in the measurements of $I(Q, \omega)$ and therefore in the determination of $\Gamma(Q)$ as a function of $Q^{2}$. The values of the diffusion coefficient $D$ reported here are compared with those measured by NMR [21] for the same range of densities and temperatures. These latter values are given with an estimated accuracy of about $\pm 10 \%$. The good agreement observed between the two sets of diffusion coefficients is important because it justifies a posteriori the applicability of the jump diffusion model and the hypothesis that rotational motions contribute only to the background. Moreover, it appears that $D$ increases strongly as the density of the medium decreases, a result in agreement with both previous experimental [21] and simulation works $[15,18,19]$.

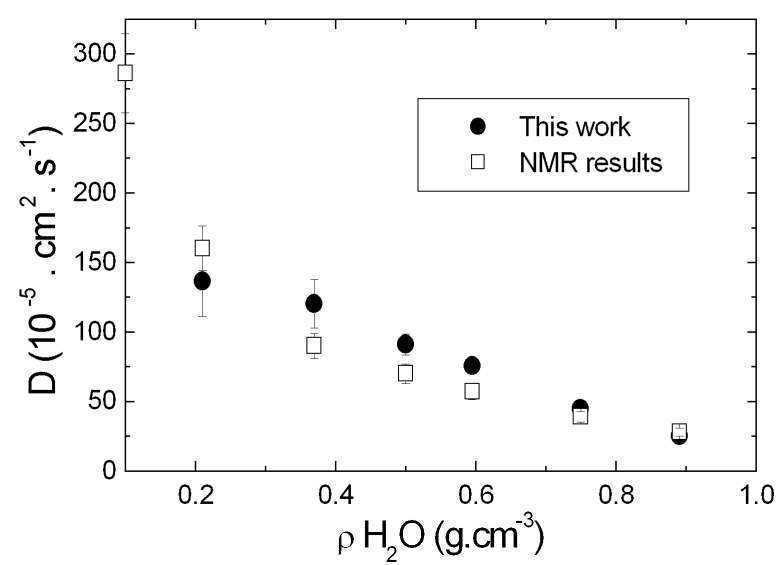

Fig. 3 Evolution of $D$ as a function of the density of water. RMN data, ref. [21] (empty square), present work (solid dots). 


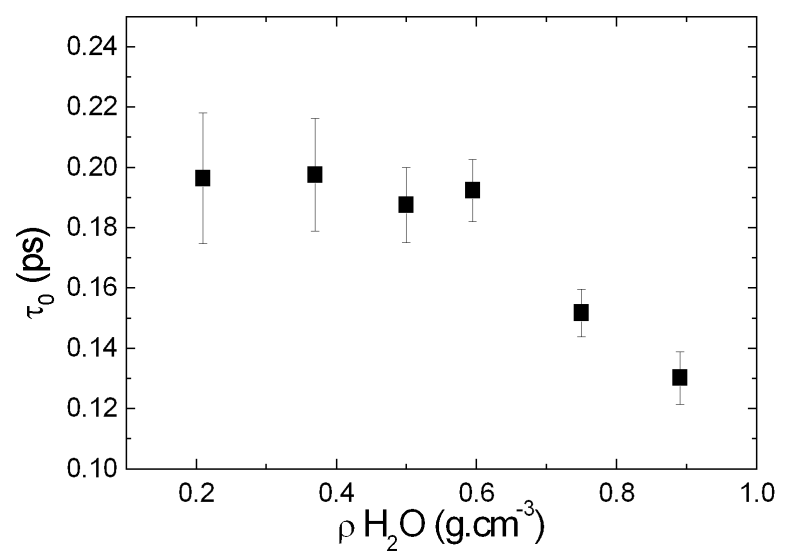

Fig. 4 Evolution of the residence time $\tau_{0}$ as a function of the density of water.

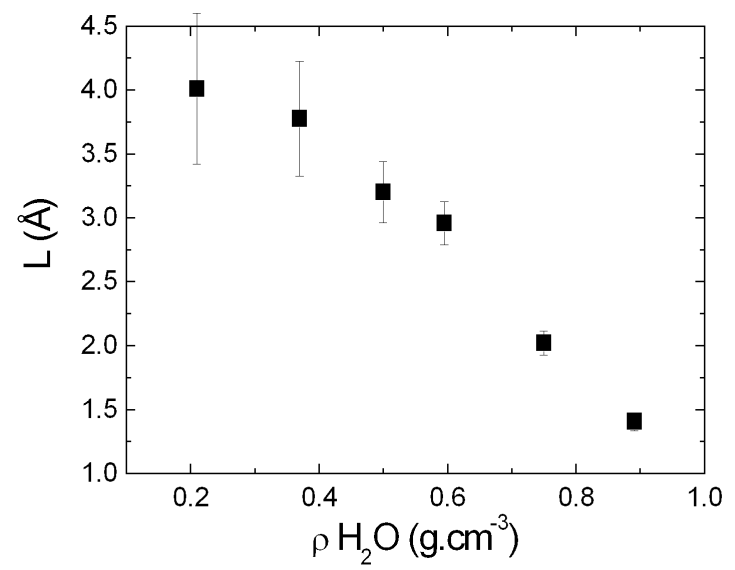

Fig. 5 Evolution of the mean jump diffusion length as a function of the density of water.

The evolution of the residence time $\tau_{0}$ is reported in Fig. 4. We observe a slight increase of $\tau_{0}$ with values ranging between 0.14 and 0.2 ps as the density of the medium decreases. The values are roughly 10 times shorter than the ones measured in ambient liquid water. The difference between the values of $\tau_{0}$ calculated at ambient conditions and that in dense SCW might be explained by the fact that at higher temperature, the kinetic energy of the medium facilitates frequent breaking of hydrogen bonds. On the other hand, the shorter decay constant calculated in dense supercritical water compared to the lowest-density state might be due to a smaller mean-free path and more frequent collisions in the high-density state. Other authors have found a similar result by using molecular dynamics simulations. Indeed, Mizan et al. [19] have calculated the hydrogen bond persistence time $\tau_{\mathrm{HB}}$ for liquid water and for SCW at $500{ }^{\circ} \mathrm{C}$ as a function of density. This persistence time $\tau_{\mathrm{HB}}$ is defined as follows:

$$
N_{\mathrm{HB}}(t)=N_{\mathrm{HB}}(0) \exp \left(-t / \tau_{\mathrm{HB}}\right)
$$

where $N_{\mathrm{HB}}(t)$ is the number of hydrogen bond at time $t$.

It has been found that $\tau_{\mathrm{HB}}$ is about $0.1 \mathrm{ps}$ for dense supercritical water at $T=500{ }^{\circ} \mathrm{C}$ and $\rho_{\mathrm{H}_{2} \mathrm{O}}=0.659 \mathrm{~g} \cdot \mathrm{cm}^{-3}$ (six times shorter than the value reported for ambient liquid water) and increases up to 0.2 ps for gaseous densities at $T=500{ }^{\circ} \mathrm{C}$ and $\rho_{\mathrm{H}_{2} \mathrm{O}}=0.115 \mathrm{~g} \cdot \mathrm{cm}^{-3}$ [19]. Thus, the evolution of the persistence time for $\mathrm{H}$-bonds, as evaluated by molecular dynamics simulations, follows exactly the 
same trend as $\tau_{0}$, with values ranging between 0.1 and $0.2 \mathrm{ps}$. On the other hand, it has been shown above that, on the average, water molecules are H-bonded to other water molecules to form dimers or trimers. In this context, we might associate the residence time $\tau_{0}$ to the H-bond lifetime, which is in very good agreement with the value of $\tau_{\mathrm{HB}}$ calculated by molecular dynamics simulation.

If we consider now the evolution of the mean jump distance $L$ as a function of density, we observe an almost linear increase in $L$ from $1.5 \AA$ at $0.9 \mathrm{~g} / \mathrm{cm}^{3}$ to $4 \AA$ at $0.2 \mathrm{~g} / \mathrm{cm}^{3}$ (see Fig. 5). The mean jump distance reported for water at $473 \mathrm{~K}$ and $0.89 \mathrm{~g} \cdot \mathrm{cm}^{-3}$ is only slightly above that reported for ambient liquid water (1.3 $\AA$ ) [20]. However, as the density of the medium decreases, the average distance between water molecules increases strongly. As a result, in order to make a new hydrogen bond with another molecule, water has to diffuse over a longer distance.

\section{ACKNOWLEDGMENTS}

The support of the University Bordeaux I (Programmes pluri-formation $n^{\circ} 971022$ et 990814) is gratefully acknowledged.

\section{REFERENCES}

1. Physical Chemistry of Aqueous Systems: Meeting the Needs of Industry, H. J. White, J. V. Sengers, D. B. Neumann, J. C. Bellows (Eds.), Begell House, New York (1995).

2. R. W. Shaw, B. Brill, A. Clifford, A. Eckert, E. U. Franck. Chem. Eng. News 69, 26 (1991).

3. Y. Garrabos, B. L. Neindre, P. Subra, F. Cansell, C. Pommier. Ann. Chim. Fr. 17, 55 (1992).

4. Y. E. Gorbaty and A. G. Kalinichev. J. Chem. Phys. 99, 5336 (1995).

5. K. Yamanaka, T. Yamaguchi, H. Wakita. J. Chem. Phys. 101, 9830 (1994).

6. M.-C. Bellissent-Funel, T. Tassaing, H. Zhao, D. Beysens, B. Guillot, Y. Guissani. J. Chem. Phys. 107, 2942 (1997).

7. T. Tassaing, M.-C. Bellissent-Funel, B. Guillot, Y. Guissani. Europhys. Lett. 42, 265 (1998).

8. A. K. Soper, F. Bruni, M. A. Ricci. J. Chem. Phys. 106, 247 (1997).

9. H. G. Kjaergaard, B. R. Henry, H. Wei, S. Lefebvre, T. Carrington, O. S. Mortensen, M. L. Sage. J. Chem. Phys. 100, 6228 (1994).

10. G. V. Bondarenko and Y. E. Gorbaty. Mol. Phys. 74, 639 (1991).

11. Y. E. Gorbaty and G. V. Bondarenko. Appl. Spectrosc. 53, 908 (1999).

12. S. S. Xantheas. J. Chem. Phys. 102, 4505 (1995).

13. D. A. Estrin, L. Paglieri, G. Corongiu, E. Clementi. J. Phys. Chem. 100, 8701 (1996).

14. B. Guillot and Y. Guissani. In Collision and Interaction-Induced Spectroscopy, Vol. 452, G. C. Tabisz and M. N. Neuman (Eds.), p. 129, Kluwer Academic, Dordrecht (1995).

15. N. Yoshii, H. Yoshie, S. Miura, S. Okazaki. J. Chem. Phys. 109, 4873 (1998).

16. N. Matubayasi, N. Nakao, M. Nakahara. J. Chem. Phys. 114, 4107 (2001).

17. C. I. Ratcliffe and D. E. Irish. J. Phys. Chem. 86, 4897 (1982).

18. J. Marti. J. Chem. Phys. 110, 6876 (1999).

19. T. I. Mizan, P. E. Savage, R. M. Ziff. In Innovations in Supercritical Fluids: Science and Technology, Vol. 608, K. W. Hutchenson and N. R. Foster (Eds.), p. 47, American Chemical Society, Washington, DC (1995).

20. J. Teixeira, M.-C. Bellissent-Funel, S. H. Chen, A. J. Dianoux. Phys. Rev. A 31, 1915 (1985).

21. W. J. Lamb, G. A. Hoffman, J. Jonas. J. Chem. Phys. 74, 6875 (1981). 